\title{
Evolution in heterogeneous environments: genetic variability within and across different grains in Tribolium castaneum
}

\author{
SARA VIA* \& JEFFREY CONNER $\dagger$ \\ Department of Entomology and Section of Ecology and Systematics, Cornell University, Ithaca, NY 14853 and \\ tDepartment of Ecology, Ethology and Evolution, University of Illinois, Champaign, IL 61820, U.S.A.
}

\begin{abstract}
The course of adaptation to heterogeneous environments is influenced by the magnitude of genetic variation for ecologically important characters within each environment and the extent of genotype $\times$ environment interaction. Using the genetic correlation between the expression of characters in different environments as a measure of genotype $\times$ environment interaction is particularly useful for evolutionary interpretation. In this study, we estimated patterns of genetic variability and cross-environment genetic correlations for pupal weight and development time in two strains of the flour beetle Tribolium castaneum in five flours (wheat with brewer's yeast, wheat, rice, corn and oat). Wheat plus yeast is the standard medium in which the strains have been reared for hundreds of generations; other flours are novel environments. The results indicated moderate levels of genetic variation within the various flours for pupal weight but not for development time. Performance varied considerably across flours, with the highest performance for both strains found in the standard medium and the poorest in oat flour. The genetic correlations of pupal weight across flours in both strains were generally not significantly different from +1 . This suggests that evolution of body size in different flours cannot proceed independently, and that improved performance in the novel flours may produce declines in fitness in the standard environment.
\end{abstract}

Keywords: Coleoptera, environmental heterogeneity, genetic correlations, genetic variation, genotype $\times$ environment interactions, novel environment.

\section{Introduction}

Organisms frequently experience heterogeneous environments in which important environmental features vary over time or space. Spatial variation in resource type and availability is particularly common. Species that utilize many different kinds of resources across their range frequently appear to be locally adapted specialists (Fox \& Morrow, 1981; Via, 1991a). However, theoretical models of evolution in spatially variable environments suggest that either generalism or local adaptation of ecologically important traits can evolve in heterogeneous environments, depending on patterns of genetic variability within and across environments, the relative frequency of the environments, the extent of gene flow and the magnitude of costs to generalism (Via \& Lande, 1985; van

*Correspondence: Department of Entomology, Comstock Hall, Cornell University, Ithaca, NY 14853, U.S.A.
Tienderen, 1991). Generalism may involve a phenotypic change when organisms enter a new environment (phenotypic plasticity), and genotypes may differ in the extent of plastic response to different environments. This genetic variation in phenotypic plasticity is known as genotype $\times$ environment interaction, and it is of particular importance in determining the course of evolution in spatially variable environments (Via \& Lande, 1985, 1987).

When genotype $\times$ environment interaction is measured as the genetic correlation between character states expressed in different environments (Falconer, 1952; Yamada, 1962; Via, 1987) it conveniently expresses the extent to which evolution of a given trait can proceed independently in different environments (Via \& Lande, 1985). Positive genetic correlations across environments often occur when environments are very similar (although they may sometimes occur between dissimilar environments). Although positive cross-environment correlations can facilitate the evolu- 
tion of generalism if selection acts in the same direction in each environment, this may be just a short-term effect and positive correlations may be constraining if environments are very dissimilar (Via \& Lande, 1985). Cross-environment correlations of zero mean that character states in two environments can evolve independently, and they correspond to the greatest long-term lack of evolutionary constraint in heterogeneous environments (Via \& Lande, 1985). Finally, negative genetic correlations in life-history traits across environments have been of particular interest to evolutionary biologists because they can be used to measure the extent of 'trade-offs' between traits of ecological importance in different environments, providing a basis for understanding the evolution of specialization (reviews in Futuyma \& Moreno, 1988; Stearns, 1992). Empirical studies to date have generally revealed that genetic correlations across environments tend either to be positive or close to zero, providing little support for the hypothesis that specialization to different resources or environments results from some fundamental genetic trade-off (reviews in Futuyma \& Moreno, 1988; Rausher, 1988; Via, 1990). However, significant negative genetic correlations across environments have been found in few studies (Fry, 1990; Via, 1991a) and recent work suggests that the paucity of estimates of negative correlations may be partially the result of some methodological problems (Fry, 1990, 1994). Therefore, the typical magnitude of genetic correlations across environments remains an open question.

Flour beetles in the genus Tribolium provide a good model system for the study of evolution in heterogeneous environments. T. castaneum is known to utilize many different flour types (Sokoloff et al., 1966a,b); however, the extent to which this polyphagy results from local adaptation to the most common flour as opposed to individually generalized resource use is currently unknown. In addition, Tribolium spp. are serious pests of stored products. Although Tribolium in granaries or flour mills may often be exposed to only one or two grain types, mills may sometimes contain many types of grain, providing the opportunity for selection in a spatially heterogeneous environment. Given an appropriate pattern of genetic variances and cross-environment covariances, this exposure to multiple flours may eventually lead to the evolution of generalized Tribolium species that could be even more serious pests on a wide array of flours.

Previous studies have revealed that development time and the number of offspring produced by $T$. castaneum depend on flour type (Sokoloff et al., $1966 \mathrm{a}, \mathrm{b})$, that strains vary in performance on the standard medium (whole wheat flour supplemented with five per cent brewer's yeast) (Soliman \& Hardin,
1971) and that there is strain $\times$ environment interaction for 14-day larval weight in different nutritional environments (Hardin et al., 1967). Previous work on the two strains studied here also revealed strain $\times$ environment interaction in pupal weight and development time over five flour environments (Via, 1991b). However, the extent of genotype $\times$ environment interaction within strains for use of different flours is unknown and the genetic correlations within strains across flours have never been estimated.

The present study was undertaken to evaluate the extent of variation within two strains of $T$. castaneum in each of five flour environments, to estimate the extent of genotype $\times$ environment interaction for pupal weight and development time within each strain, and to determine the pattern of genetic correlations across flours for each strain. We also compared the matrices of crossenvironment genetic correlations for the two strains to evaluate the possibility that the strains differ in their genetic architecture.

\section{Materials and methods}

\section{Strains}

The two strains used in these experiments were 'Chicago cSM' (provided by Dr Michael Wade, University of Chicago) and 'Purdue ${ }^{+2}$ ' (provided by $\mathrm{Dr}$ A. E. Bell, Purdue University). Details of the synthesis of these strains can be found in Via (1991b). Because both strains have been reared in the standard medium (whole wheat flour with 5 per cent brewer's yeast, henceforth W5) for hundreds of generations (Via, 1991b), it is reasonable to suppose that they are better adapted to that medium than to the other flours tested here, which have not previously been experienced and which may therefore be considered as novel environments.

\section{Experimental design}

Separate half-sib analyses (Falconer, 1989) were conducted on the two strains. Before each analysis, an oviposition screen was performed to eliminate unmated females and males with low mating ability in an attempt to produce a roughly balanced dataset. For these screens, 30 males were each mated to six females in $8 \mathrm{~g}$ of W5 for several days. All beetles were chosen randomly from large stock cultures. Each male was marked with a small dab of white paint on the elytra, and females in each vial were also individually marked with different colours. Preliminary experiments revealed that this marking procedure did not interfere with mating or oviposition. After the mating period, 
each female was transferred to an individual vial containing fine-sifted flour and allowed to oviposit for $24 \mathrm{~h}$, after which eggs were sifted from the flour and counted. Females with fewer than five eggs and males with more than two such females were eliminated, along with enough others chosen at random to produce the final designs. Very few individuals were actually eliminated for this reason, although, even this slight selection may cause our estimates of genetic variation to be conservative. For Chicago, the design was 20 sires $\times 4$ dams $/$ sire $\times 5$ flours $\times 5$ replicates $=2000$ offspring over two time blocks. The design was the same for Purdue, except that six progeny instead of five were tested within each flour (2400 offspring).

Trials were initiated by a series of ovipositions by the mated females. For both strains, oviposition into individual vials of fine-sifted flour occurred every other day for $24 \mathrm{~h}$, after which females were returned to their mating vial for 1 day. Because counting and allocating the eggs to the rearing vials was extremely time consuming, females were divided into two groups, with two females/male in each group. Groups were on alternate day schedules. For Chicago, oviposition occurred into the same type of flour as that used for the larval rearing, and flours were experienced by each female in random order (with W5 first for all females for logistical reasons). After $24 \mathrm{~h}$ of oviposition, eggs were sifted from the oviposition vials and two eggs were allocated to either two or three replicate vials containing $1.2 \mathrm{~g}$ of the same flour (two replicates in block 1, three replicates in block 2). For Purdue, all oviposition occurred into W5, and in each oviposition period, one egg was allocated to a vial of each of the five flours $(1.4 \mathrm{~g})$. This greatly simplified the oviposition procedure. For both strains, test vials were then randomized and placed in an incubator at $30^{\circ} \mathrm{C}$ and 90 per cent $\mathrm{RH}$ for rearing. At pupation, pupae were sifted out of the flour, sexed, returned to empty vials and checked three times daily for eclosion to determine development times. Pupal weights were taken on approximately the third day after pupation, using a Cahn Model 28 Ultramicrobalance.

\section{Data analysis}

All analyses were performed separately on each strain. To study genetic variance in pupal weight and development time within each environment, separate analyses of variance were performed for each of the two traits within each flour using PROC GLM in SAS (SAS Institute, 1985). Sex of the offspring was considered a fixed effect, with Block, Sire, Group (nested within Sire), and Dam (nested within Group and Sire) as random effects. The main effect of Group was not significant in any of the models, nor were any of the interactions containing
Group, so Group was dropped from all subsequent models. The initial models included all possible twoand three-way interactions. These interactions were not of biological interest, but were included to check for possible significant effects. The nonsignificant interactions were eliminated and the models were recalculated.

The proportions of total phenotypic variance accounted for by each of the effects in the final models were estimated using restricted maximum likelihood methods (Shaw, 1987), employing the REML option in PROC VARCOMP in SAS (SAS Institute, 1985). In eight of 24 cases the iterative model did not converge with the Block main effect included, so these were re-run on the residuals of an ANOVA with Block as the only effect; the REML models then converged in all eight cases. Narrow-sense heritabilities were calculated as four times the Sire variance component divided by the total variance (Falconer, 1989). Significance levels for the heritabilities were taken from those of the corresponding Sire terms in the single-flour ANOVAs.

Genetic variance for the two traits across the five environments was examined by combining the data from all five flours in a single ANOVA for each strain and trait. As none of the interactions in the single-flour models was significant in more than two of the 20 single-flour ANOVAs, these interactions were not included in the five-flour models. The four two-way interactions between flour and the other main effects were included in the first run of the models; as above, interactions that were not significant and not of biological interest were eliminated and the models were recalculated.

The pattern of genetic variability across environments can be studied by estimating either the genotype $\times$ environment interactions or the genetic correlations among single traits expressed in different environments (Yamada, 1962; Via, 1984, 1987). The magnitudes of genotype $\times$ environment interaction pooled over all the flours can be seen in the five-flour ANOVAs. However, a more detailed set of analyses using all pairwise two-flour models is required to know about the pattern of genotype $\times$ environment interaction in more detail. These pairwise ANOVAs are also very useful in estimating and testing the significance of the cross-environment genetic correlations (Fry, 1992).

The genetic correlations across pairs of environments are potentially more useful for evolutionary interpretation than is the magnitude of the genotype $\times$ environment interaction (for examples, see Via, 1987). Falconer (1952) first described how a character expressed in different environments could be considered as a group of 'character states', each of which has some genetic variance in the environment in 
which it is expressed, and which are potentially but not necessarily genetically correlated. These 'crossenvironment' genetic correlations are useful because they provide an estimate of the extent to which evolution in different environments can proceed independently.

The genetic correlations of pupal weight and development time across the five flours were calculated in three different ways. Firstly, we calculated the Pearson product-moment correlations among half-sib family means in the five environments. Although these are biased by within-family environmental variance and covariance, the magnitude of this bias is divided by the number of offspring in each half-sib family (Via, 1984 ) and the number of dams mated to each sire (in this case, 20-24 and 4, respectively). This bias is reduced as the heritability increases, but the general expectation is that cross-environment correlations of family means will be less than correlations estimated using variance components (J. D. Fry, personal communication). The advantages of using half-sib family means are that the correlations can be tested for differences from zero like any Pearson coefficient, and covariance matrices of half-sib means can be easily compared (see below).

Secondly, variance component correlations across environments were calculated in two ways using the methods of Fry (1992), which involve estimating the Sire component of covariance across the flours as the variance component from the 'Sire' main effect in a two-environment ANOVA. The 'Sire' variance components in this set of all possible two-environment ANOVAs were estimated in PROC VARCOMP of SAS using both the method of equating expected and observed mean squares (henceforth called the TYPE1 estimates, because they were made using Type 1 (sequential) sums of squares), and by restricted maximum likelihood (REML). Fry (1992) mentions that the TYPE1 estimates may be biased if the dataset is unbalanced. He also cautions that because many REML algorithms constrain the variance components to be positive, the use of REML may be unwise if the genetic covariance across environments is suspected to be negative. Here, we found from the correlations of family means and the TYPE 1 estimates that only three of 40 correlations were nominally negative, and none was significantly less than zero. Thus, in our case (the dataset was slightly unbalanced) the REML estimates of the additive genetic covariance across environments should generally be an improvement over the TYPE1 estimates.

Because Fry's (1992) methods have not, to our knowledge, been used in other empirical studies of cross-environment correlations, we present all three methods of estimation for comparison. These correlations and the associated two-environment ANOVAs are used here to test two different biological hypotheses. (1) Do the cross-environment genetic correlations differ from zero? A correlation of zero across environments corresponds to complete genetic independence between two character states. This was tested using the significance values for the Sire main effect in the two-environment ANOVAs, and was compared with the test for significance of the family mean correlations, which tests the same hypothesis. (2) Do the cross-environment correlations differ from +1 ? A correlation of +1 corresponds to complete genetic dependence between two character states, and means that their evolution is necessarily coupled. This was tested using the Sire $\times$ Flour interaction in the twoflour ANOVAs; a significant interaction indicates that the cross-environment genetic correlation is $<+1$.

Simple product-moment correlations of family means were used to derive a qualitative picture of the genetic correlations between the two traits within each flour and the genetic correlations between the sexes across each flour. The latter provide estimates of the potential for the evolution of sexual dimorphism (Lande, 1980).

Given the differences between strains in the average performance across flours seen here and elsewhere (Via, 1991b), the genetic variance/covariance matrices of the two strains were compared to see if the strains may also differ in genetic architecture. A likelihood ratio test of the homogeneity of covariance matrices was performed, using the covariance matrices of family means (PROC DISCRIM, SAS Institute, 1985). Both the $10 \times 10$ matrices (two traits expressed in five environments) and the $5 \times 5$ matrices for each trait separately were compared.

\section{Results}

The five flours definitely differed in their suitability for Tribolium growth, as reflected in the reaction norms constructed by plotting relative mean pupal weights and development times across flours (Fig. 1a, b). The narrow-sense heritabilities and evolvabilities (Houle, 1992) of the two traits estimated within each flour (Table 1) show that there was moderate additive genetic variation for pupal weight in most environments but much less genetic variation for development time was seen for both strains. Given the reaction norms (Fig. 1), it is not surprising that the ANOVA across all flours showed significant variability among beetles raised in different flours ('Flour' main effect; Table 2). This effect was particularly strong for development time where over 85 per cent of the total 
phenotypic variance was attributable to flour type in both strains. The five-flour ANOVAs also reveal that there was significant genotype $\times$ environment interaction for development time in the Chicago strain and for pupal weight in the Purdue strain (Flour $\times$ Sire, Table 2).
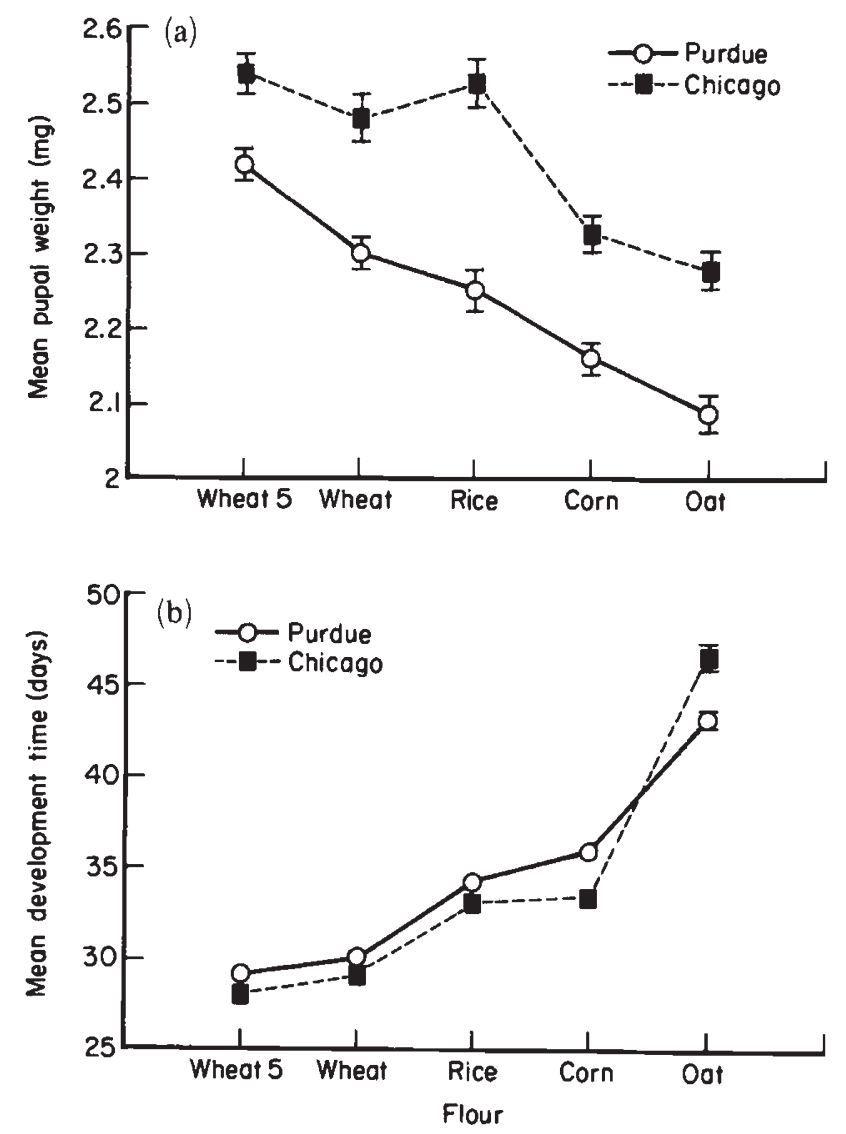

Fig. 1 Reaction norms of the two traits across the five flours for both strains of Tribolium castaneum. Means \pm two standard errors of the mean are plotted. (a) Pupal weight. (b) Development time. Note that for development time, the error bars are too small to see in all flours except Oat.
The cross-environment genetic correlations among the traits expressed in the different flours describe the potential for independent evolution of the traits in different environments (Tables 3 and 4). Note that in all cases, the correlations of family means were less than the estimates of the additive genetic correlations made using the variance component method of Fry (1992), as expected given the bias of the family mean method as a result of inclusion of variance and covariance attributable to the environment and common dams (J. D. Fry, personal communication).

All of the genetic correlations among pupal weights in the different environments were positive for both strains. However, using either the test for the family mean correlations or the Sire effect in the relevant twoflour ANOVAs, only seven of 10 correlations in Chicago and four of 10 correlations for Purdue were significantly greater than 0 (at an individual level of $P<0.01$, using a sequential Bonferroni procedure). In general, although the values of the correlations estimated by family means and variance components differed considerably, the qualitative picture of significance provided by tests of the product-moment correlations is very consistent with that provided by the two-factor ANOVAs (Tables 3 and 4).

In the Chicago strain, the genetic correlations among pupal weights were high for most flours, except between W5 and oat (Table 3a). This is not surprising, given that the reaction norms reveal that these were the two most different environments (Fig. 1). In the Purdue strain, there were very strong positive correlations among pupal weight in wheat 5 , wheat and corn, with weaker correlations among pupal weight expressed in the other flours, again suggesting that correlations are lowest between traits expressed in environments that were the most different.

The genetic correlations among development times were lower overall and more variable than those for pupal weight, with a few (nonsignificant) negative

Table 1 Measures of genetic variability for pupal weight and development time for the two strains of Tribolium castaneum: narrow sense heritability $\left(h_{\mathrm{N}}^{2}\right)$ and coefficient of additive genetic variability (evolvability, $\left(\mathrm{V}_{\mathrm{A}}\right)$. Significance levels for the heritabilities are from the Sire main effect in single-flour ANOVAs

\begin{tabular}{|c|c|c|c|c|c|c|c|c|c|c|c|}
\hline & & \multicolumn{2}{|c|}{ W5 } & \multicolumn{2}{|c|}{ Wheat } & \multicolumn{2}{|c|}{ Rice } & \multicolumn{2}{|c|}{ Corn } & \multicolumn{2}{|c|}{ Oat } \\
\hline & & $h_{\mathrm{N}}^{2}$ & $\mathrm{CV}_{\mathrm{A}}$ & $h_{\mathrm{N}}^{2}$ & $\mathrm{CV}_{\mathrm{A}}$ & $h_{\mathrm{N}}^{2}$ & $\mathrm{CV}_{\mathrm{A}}$ & $h_{\mathrm{N}}^{2}$ & $\mathrm{CV}_{\mathrm{A}}$ & $h_{\mathrm{N}}^{2}$ & $\mathrm{CV}_{\mathrm{A}}$ \\
\hline \multirow{2}{*}{$\begin{array}{l}\text { Pupal weight } \\
\text { (mg) }\end{array}$} & Chicago & $0.23^{*}$ & 2.45 & 0.20 & 2.42 & 0.35 & 2.99 & $0.64^{* * *}$ & 3.60 & $0.68^{* * *}$ & 3.90 \\
\hline & Purdue & 0.37 & 7.54 & $0.50^{* * *}$ & 3.27 & $0.41^{* *}$ & 3.84 & $0.48^{* * *}$ & 3.22 & 0 & 0 \\
\hline \multirow{2}{*}{$\begin{array}{r}\text { Development } \\
\text { time (days) }\end{array}$} & Chicago & 0.18 & 0.89 & 0.32 & 1.59 & 0.10 & 0.84 & 0 & 0 & $0.36^{*}$ & 3.79 \\
\hline & Purdue & 0.04 & 0.38 & 0.13 & 0.67 & 0 & 0 & $0.28^{*}$ & 1.31 & 0 & 0 \\
\hline
\end{tabular}

${ }^{*} P \leqslant 0.05,{ }^{* *} P \leqslant 0.01,{ }^{* * *} P \leqslant 0.001$. 


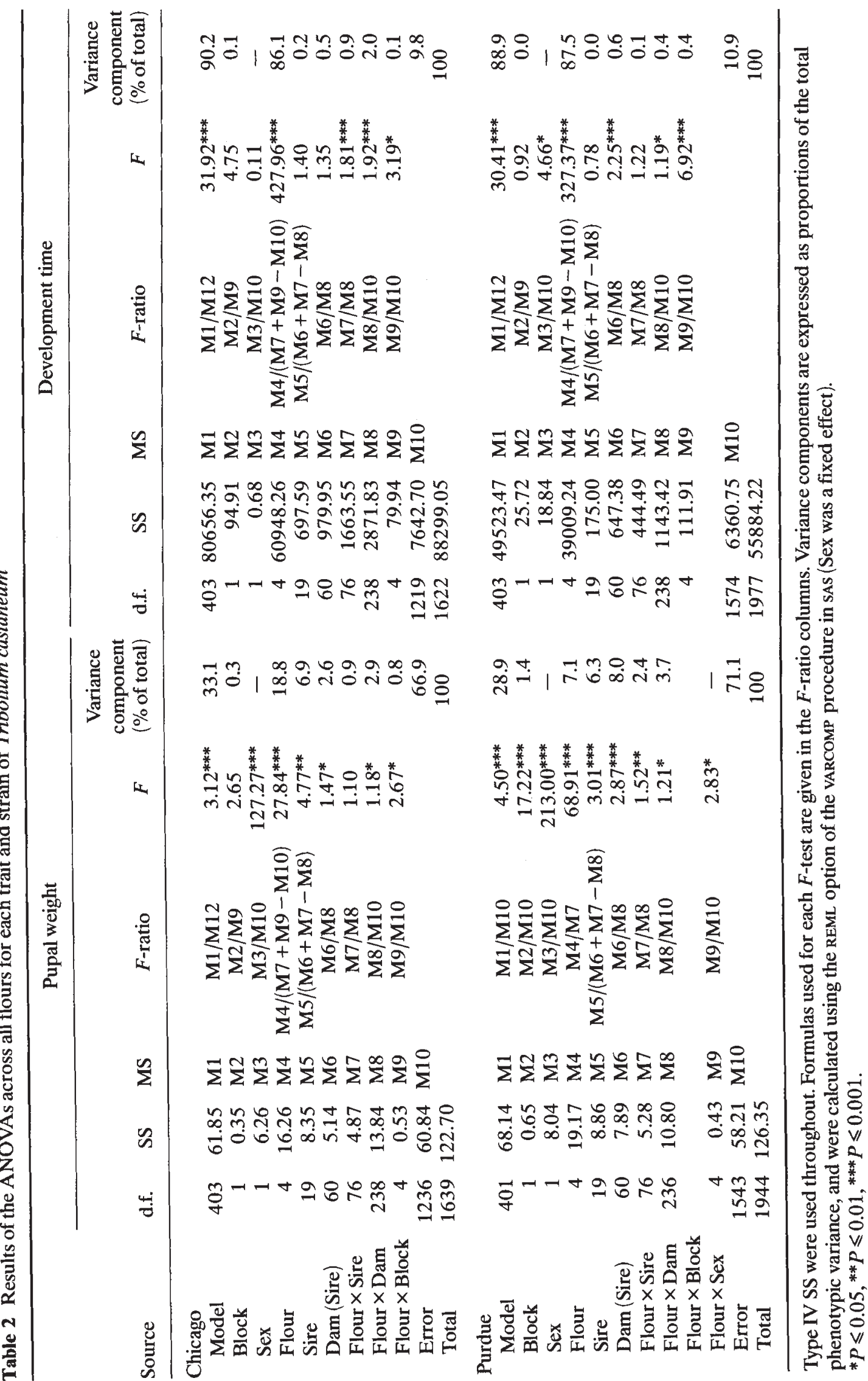


Table 3 Cross-environment correlations for pupal weight in Tribolium castaneum

\begin{tabular}{|c|c|c|c|c|c|}
\hline & Wheat & Rice & Corn & Oat & Method \\
\hline \multicolumn{6}{|c|}{ (a) Pupal weight, Chicago strain } \\
\hline \multirow[t]{4}{*}{ W5 } & $0.02 / N S$ & $0.005 / N S$ & $0.02 / N S$ & $0.07 / 0.004$ & \\
\hline & 1.13 & 1.04 & 0.90 & 0.41 & TYPE1 \\
\hline & 1.03 & 1.01 & 0.84 & & REML \\
\hline & $0.66(0.001)$ & $0.63(0.003)$ & $0.51(0.02)$ & $0.37(\mathrm{NS})$ & FamMean \\
\hline \multirow[t]{4}{*}{ Wheat } & & $0.008 / N S$ & $0.0001 / N S$ & $0.0002 / N S$ & \\
\hline & & 1.49 & 1.36 & 1.22 & TYPE1 \\
\hline & & 1.15 & 1.06 & 1.05 & REML \\
\hline & & $0.59(0.006)$ & $0.53(0.01)$ & $0.57(0.008)$ & FamMean \\
\hline \multirow[t]{4}{*}{ Rice } & & & $0.002 / N S$ & $0.01 / N S$ & \\
\hline & & & 1.07 & 0.92 & TYPE1 \\
\hline & & & 0.95 & 0.78 & REML \\
\hline & & & $0.66(0.002)$ & $0.49(0.03)$ & FamMean \\
\hline \multirow[t]{4}{*}{ Corn } & & & & $0.0001 / N S$ & \\
\hline & & & & 1.12 & TYPE1 \\
\hline & & & & 0.98 & REML \\
\hline & & & & $0.71(0.0004)$ & FamMean \\
\hline \multicolumn{6}{|c|}{ (b) Pupal weight, Purdue strain } \\
\hline \multirow[t]{4}{*}{ W5 } & $0.001 / N S$ & $0.06 / 0.025$ & $0.0001 / N S$ & $N S / 0.03$ & \\
\hline & 1.04 & 0.55 & 1.22 & 0.19 & TYPE1 \\
\hline & 1.22 & 0.63 & 1.28 & - & REML \\
\hline & $0.75(0.0001)$ & 0.33 (NS) & $0.75(0.0002)$ & 0.25 (NS) & FamMean \\
\hline \multirow[t]{4}{*}{ Wheat } & & $0.04 / 0.02$ & $0.0001 / N S$ & $0.02 / N S$ & \\
\hline & & 0.45 & 1.31 & 0.61 & TYPE1 \\
\hline & & 0.46 & 1.11 & - & REML \\
\hline & & 0.41 (NS) & $0.85(0.0001)$ & $0.50(0.02)$ & FamMean \\
\hline \multirow[t]{4}{*}{ Rice } & & & $0.007 / N S$ & $N S / 0.07$ & \\
\hline & & & 0.78 & 0.39 & TYPE1 \\
\hline & & & 0.75 & & REML \\
\hline & & & $0.59(0.006)$ & $0.22(\mathrm{NS})$ & FamMean \\
\hline \multirow[t]{4}{*}{ Corn } & & & & $0.06 / N S$ & \\
\hline & & & & 0.57 & TYPE1 \\
\hline & & & & $\overline{0} 36(\mathrm{NS})$ & REML \\
\hline & & & & $0.36(\mathrm{NS})$ & FamMean \\
\hline
\end{tabular}

Each cell in the table has four lines. The first (italicized) gives significance values of the correlation for two tests from the twoenvironment ANOVAs: before the solidus is the test of whether the correlation differs from zero (from the Sire main effect of the ANOVA); after the solidus is the test of whether the correlation differs from +1 (from the Sire $\times$ Flour interaction in the ANOVA). The second and third lines in each cell are the two variance component estimates of the correlation from the twoenvironment ANOVAs, the first using TYPE1 estimates and the second using REML estimates of the (co)variance components. The fourth line in each cell is the family mean correlation with its significance value, testing whether it is different from zero. Individual significance values greater than 0.10 are listed as NS, but only those values $\leq 0.01$ are significant over all correlations within each strain and test using a sequential Bonferroni correction (Rice, 1989). Missing values (indicated by a dash) are for trait combinations for which one of the variance components in the two-environment ANOVA was zero (sometimes this occurred only for the REML estimates).

values (Table 4). However, because of low or nonsignificant genetic variances, these estimates are probably not very reliable and many of the variance component correlations for development time in the different flours could not even be estimated because one or both variance components were equal to zero (particularly in the Purdue strain). The strongest cross-environment correlations were between wheat and wheat 5 for both strains, and wheat 5 and rice for Chicago, again reflecting the apparent levels of similarity of these environments. 
Table 4 Cross-environment correlations for development time in Tribolium castaneum

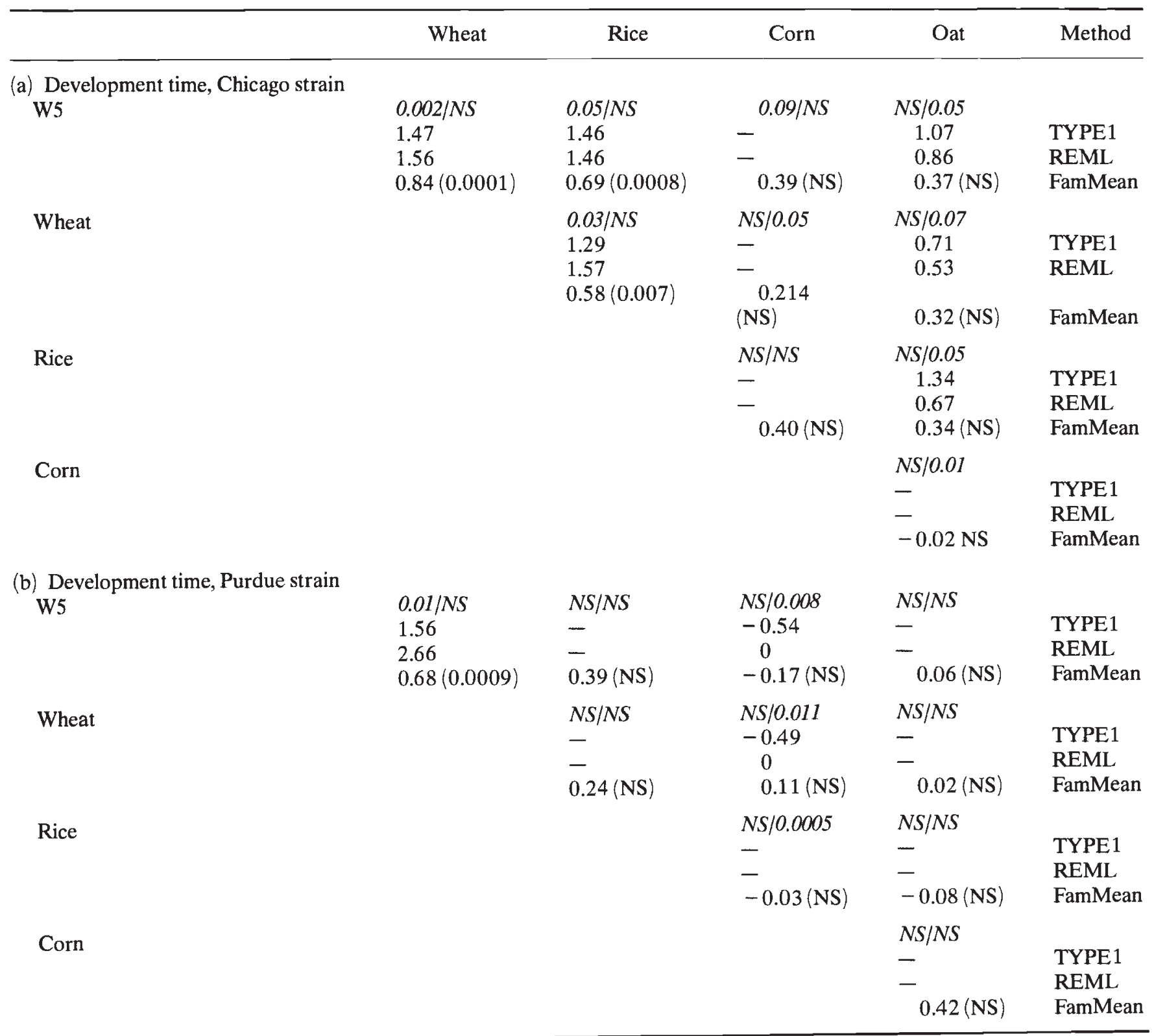

See Table 3 footnote for details.

Individual significance values greater than 0.10 are listed as NS, but only those values $\leq 0.007$ are significant over all correlations within each strain and test using a sequential Bonferroni correction.

The genetic correlations between pupal weight and development time within each flour (Table 5) show that the traits were essentially uncorrelated and could thus potentially evolve independently within each of these five environments. There were possible positive correlations between the traits within wheat and oat for the Chicago strain, but correcting for multiple comparisons, these were not statistically significant.

The genetic correlations between the sexes for each trait within each flour (Table 6) were all low to moderately positive, with significant correlations scattered across all strains, traits and flours. There seems to be no clear pattern here, although it is somewhat surprising how low these correlations are in general.

Comparing the $10 \times 10$ genetic variance/covariance matrices (two traits in five environments) of the two strains suggests that the matrices may be different $\left(\chi_{55}^{2}=70.4, P=0.08\right)$. Analyses of the $5 \times 5$ matrices for the two traits separately show that the pupal weight matrices are not significantly different $\left(\chi_{15}^{2}=21.1\right.$, $P=0.13$ ), but the development time matrices are different $\left(\chi_{15}^{2}=34.7, P<0.005\right)$. 
Table 5 Genetic correlations between pupal weight and development time of Tribolium castaneum within each of five flours (correlations of family means)

\begin{tabular}{lcr}
\hline & Chicago & Purdue \\
\hline W5 & 0.058 & -0.026 \\
& $(0.80)$ & $(0.91)$ \\
Wheat & 0.463 & 0.339 \\
& $(0.04)$ & $(0.14)$ \\
Rice & 0.358 & 0.248 \\
& $(0.12)$ & $(0.29)$ \\
Corn & -0.218 & 0.135 \\
& $(0.36)$ & $(0.57)$ \\
Oat & 0.443 & 0.338 \\
& $(0.05)$ & $(0.14)$ \\
\hline
\end{tabular}

Values in parentheses are $P>0$. Individual $P$ values must be $\leq 0.01$ to be significant at $P \leq 0.05$ within strains using a sequential Bonferroni correction.

Table 6 Genetic correlations across the sexes of Tribolium castaneum in each of five flours (correlations of family means)

\begin{tabular}{lccccc}
\hline & \multicolumn{2}{c}{ Pupal weight } & & \multicolumn{2}{c}{ Development time } \\
\cline { 2 - 3 } \cline { 5 - 6 } & Chicago & Purdue & & Chicago & Purdue \\
\hline \multirow{2}{*}{ W5 } & 0.597 & 0.315 & & 0.617 & 0.049 \\
& $(0.005)$ & $(0.17)$ & & $(0.004)$ & $(0.83)$ \\
Wheat & 0.366 & 0.592 & & 0.602 & 0.436 \\
& $(0.11)$ & $(0.006)$ & & $(0.005)$ & $(0.05)$ \\
Rice & 0.425 & 0.567 & & 0.428 & 0.167 \\
& $(0.06)$ & $(0.009)$ & & $(0.06)$ & $(0.48)$ \\
Corn & 0.653 & 0.534 & & -0.111 & 0.576 \\
& $(0.002)$ & $(0.01)$ & & $(0.64)$ & $(0.008)$ \\
Oat & 0.526 & 0.080 & & 0.661 & 0.095 \\
& $(0.02)$ & $(0.74)$ & $(0.002)$ & $(0.69)$ \\
\hline
\end{tabular}

Values in parentheses are $P>0$. Individual $P$ values must be $\leq 0.01$ to be significant at $P \leq 0.05$ within each strain/trait combination using a sequential Bonferroni correction.

\section{Discussion}

We found that W5, wheat, rice, corn and oat flours clearly constitute different environments for Tribolium castaneum. Not surprisingly, performance (measured by either pupal weight or development time) was highest in the standard medium in which both strains have been raised for several hundred generations (Fig. 1a, b). For both strains, performance was lower in the novel environments and was particularly poor in oat flour. It is of interest to note that we have previously shown that cannibalistic behaviour is enhanced in oat flour relative to the standard medium (Via, 1991). Larval cannibalism appears to rescue individuals reared in oat flour from its unsuitable nutritional effects, lowering development time to approximately the same level as seen in W5 (S. Via, unpublished data). Because the experimental design used here precluded the opportunity for cannibalism, the poor quality of the novel environments could be fully observed.

Significant genetic variability in pupal weight was seen in three of five flours for both strains. Both strains showed high heritabilities in corn flour. The Chicago strain showed a considerable heritability in oat flour whereas no genetic variance could be detected within Purdue in that environment. This may be because pupal weight of Purdue in oat was at the lowest level that is consistent with successful eclosion to the adult stage, and variance is often diminished when populations are at a lower or upper bound. The $\mathrm{CV}_{\mathrm{A}}$ s provide a picture that is roughly consistent with the estimated heritabilities and significance levels from the Sire effects in the single-flour ANOVAs, except for pupal weight in Purdue in W5, where the $\mathrm{CV}_{\mathrm{A}}$ was relatively large, but the heritability was not significant, possibly due to high environmental variance.

The general absence of significant additive genetic variation in development time for both strains suggests that there may be little potential for evolution of reduced development time in the novel flours, even though it took both strains 3-5 days longer to develop in most of the novel flours than it did in the standard medium (W5). Notably, the only significant genetic variance for development time was seen in the Chicago strain in oat flour, where development time was retarded by almost 20 days. However, there was no detectable genetic variance in this same poor flour within the Purdue strain. When interpreting data for significance of genetic variances for both characters, it is worth noting that failing to estimate a statistically significant component of variance does not mean that variance is necessarily absent. Very low levels of genetic variation can permit response to selection but may not be significantly greater than zero given a moderate sample size (Mitchell-Olds \& Bergelson, 1990).

The generally high pattern of genetic correlations in pupal weight across environments (all but one not significantly different from +1 , Table 3 ) suggests that independent evolution of body size in the novel flours in unlikely. Thus, if pupal weight is under stabilizing selection, as is typical for quantitative traits of ecological importance, improvement in pupal weight in a novel flour would be expected to produce a correlated increase in size in the standard medium, potentially 
resulting in a decrease in fitness. This suggests that local adaptation and specialization to different grains might be more likely in Tribolium castaneum than is the evolution of generalized resource use. It would be very interesting to examine reaction norms for pupal weight for populations from granaries containing several flour types in order to test this hypothesis.

Little genetic variance was seen for development time, which reduces our ability to estimate the crossenvironment genetic correlations. Few of the correlations were significantly different from zero when corrected for multiple comparisons, and many were not different from either zero or one. This probably indicates the low level of reliability of these estimates, given a fairly small sample size within each flour and very low genetic variances, and they provide a useful illustration of the difficulty of obtaining precise estimates of genetic correlations. Although the sample size used here $(20$ sires $\times 4$ dams $\times 5-6$ replicates per flour) is too small for accurate estimation of genetic correlations for characters with low genetic variability, note that 2000-2400 offspring were measured in each sib analysis. Thus, logistical considerations are likely to prevent many evolutionary biologists from tackling experimental designs of a much greater magnitude when evaluation of multiple environments is the goal. This may limit our understanding of the potential for evolution in heterogeneous environments that consist of more than two or three patch types.

Genetic correlations between character states expressed in different environments are pivotal to understanding the potential for evolution in populations that experience multiple environments. Such correlations have been historically difficult to estimate because the character states are not measured on the same individuals. Various different methods have been used to complement the simple but biased method of using the correlations of family means in different environments. Until recently, methods emphasized the statistical relationship between genotype $\times$ environment interaction and the cross-environment correlation (Falconer, 1952; Yamada, 1962; Fernando et al. 1984). Fry's (1992) method for using the Sire main effect in two-environment ANOVAs represents a real advance in the statistics of cross-environment correlations because these correlations are relatively easy to estimate using standard computer packages such as sAs, and the two-environment ANOVAs provide obvious and simple tests of the two relevant hypotheses: is the cross-environment genetic correlation in question different from zero (if not, there is complete genetic independence of evolution in the two environments), and is the correlation different from +1 (if not, there is complete genetic dependence between the character states)? Tests of these hypotheses are accomplished using the Sire main effect (tests for a difference from zero) and the Sire $\times$ Environment interaction (tests for a difference of the correlation from +1 ) in the two-flour ANOVAs. However, Fry's methods can have problems when data are unbalanced and correlations across environments are suspected to be negative. This is because REML methods, which produce unbiased estimates of variance components for unbalanced data, are usually constrained to be positive.

Here, family mean correlations across environments were compared with variance component estimates using Fry's method using two different algorithms to estimate the variance components (ANOVA TYPE1 and REML). In general, the correlations of half-sib family means underestimated the genetic correlations as reflected in the variance component correlations. This has also been observed previously in other empirical studies (Via, 1984, 1991a) and is as expected by Fry on theoretical grounds (J. D. Fry, personal communication). However, the frequent occurrence of variance component correlations appreciably greater than one is unsettling and suggests that, for low heritabilities, sampling variance in datasets of this size is so large that precise estimation is impossible. Comparison of the TYPE1 and REML estimates for the crossenvironment correlations of pupal weight in the Chicago strain suggests that the REML estimates behave more reasonably, because they are generally not so much greater than +1 . Given the slight inbalance in the data, this is what we expected. However, this pattern of more reasonable behaviour does not hold for the other strain or the other character. Even though the family mean correlations are known to be biased downward, particularly for low heritability, they provided a generally very accurate picture of whether these cross-environment correlations were different from zero. This did not differ from conclusions that could be drawn from the test of the same hypothesis using the two-environment ANOVAs.

The general instability of the estimates of the variance component correlations suggests that the use of the qualitative picture provided by the crossenvironment correlations of half-sib family means, accompanied by tests of significant difference from zero and one from the two-environment ANOVAs may prove to be the most reliable indicators of the extent of genetic dependence of a set of character states in different environments. Estimates of crossenvironment genetic correlations that are precise enough to be used for predictive purposes will be much more difficult to obtain and are probably not necessary for most evolutionary studies. 


\section{Acknowledgements}

We are grateful to Susan Huckle and Elizabeth Ayesse for excellent technical assistance. Stock cultures of Tribolium and advice on rearing procedures were kindly supplied by Michael Wade and A. E. Bell (and associates). Support for this study and the preparation of the manuscript was provided by NIH (GM34523), a Searle Scholars Award and NSF grant (DEB9207572) to S.V.

\section{References}

FALCONER, D. S. 1952. The problem of environment and selection. Am. Nat., 86, 293-298.

FALCONER, D. S. 1989. Introduction to Quantitative Genetics, 3rd edn. John Wiley and Sons, New York.

FERNANDO, R. L., KNIGHTS, S. A. AND GIANOLA, D. 1984. On a method of estimating the genetic correlation between characters measured in different experimental units. Theor. Appl. Genet., 67, 175-178.

FOX, L. R. AND MORROW, P. A. 1981. Specialization: species property or local phenomenon? Science, 211, 887-891.

FRY, J. D. 1990. Trade-offs in fitness on different hosts: evidence from a selection experiment with a phytophagous mite. Am. Nat., 136, 569-580.

FRY, J. D. 1992. The mixed-model analysis of variance applied to quantitative genetics: biological meaning of the parameters. Evolution, 46, 540-550.

FRY, J. D. 1994. The 'general vigor' problem: can antagonistic pleiotropy be detected when genetic covariances are positive? Evolution, 47, 327-333.

FUTUYMA, J. D. AND MORENO, G. 1988. The evolution of ecological specialization. Ann. Rev. Ecol. Syst., 19, 207-234.

HARDIN, R. T., ROGLER, J. C. AND BELL, A. E. 1967. Genetic and environmental interactions in growth of Tribolium castaneum. Can. J. Zool., 45, 139-144.

HOULE, D. 1992. Comparing evolvability and variability of quantitative traits. Genetics, 130, 195-204.

LANDE, R. 1980. Sexual dimorphism, sexual selection and adaptation in polygenic characters. Evolution, 34, 292-305.

MITCHELL-OLDS, T. AND BERGELSON, J. 1990. Statistical genetics of an annual plant, Impatiens capensis. I. Genetic basis of quantitative variation. Genetics, 124, 407-415.

RAUSHER, M. D. 1988. Is coevolution dead? Ecology, 69, 898-901.

RICE, W. R. 1989. Analyzing tables of statistical tests. Evolution, 43, 223-225.
SAS INSTITUTE. 1985. SAS Users Guide: Statistics. SAS Institute, Cary, NC.

SHAW, R. 1987. Maximum-likelihood approaches applied to quantitative genetics of natural populations. Evolution, 41, 821-826.

SOKOLOFF, A., FRANKLIN, I. R. AND LAKHANPAL, R. K. 1966 a. Comparative studies with Tribolium. II. Productivity of $T$. castaneum Herbst and T. confusum Duval on natural semi-synthetic and synthetic diets. J. Stored Prod. Res., 1, 313-324.

SOKOLOFF, A., FRANKLIN, I. R., OVERTON, L. F. AND Ho, F. K. 1966 b. Comparative studies with Tribolium (Coleoptera: Tenebrionidae). I. Productivity of $T$. castaneum Herbst and $T$. confusum Duval on several commercially available diets. J. Stored Prod. Res., 1, 295-311.

SOLIMAN, M. H. AND HARDIN, R. T. 1971. Variation in populations of Tribolium castaneum Herbst (Coleoptera: Tenebrionidae). II. Developmental rate and productivity. J. Stored Prod. Res., 8, 1-10.

STEARns, s. c. 1992. The Evolution of Life Histories. Oxford University Press, NY.

VAN TIENDEREN, P. H. 1991. Evolution of generalists and specialists in spatially heterogeneous environments. Evolution, 45, 1317-1331.

VIA, s. 1984. The quantitative genetics of polyphagy in an insect herbivore. II. Genetic correlations in larval performance within and across host plants. Evolution, 38, 896-905.

VIA, s. 1987. Genetic constraints on the evolution of phenotypic plasticity. In: Loeschke, V. (ed.) Genetic Constraints on Adaptive Evolution, pp. 47-71. Springer, Berlin.

VIA, s. 1990. Ecological genetics and host adaptation in herbivorous insects: the experimental study of evolution in natural and agricultural systems. Ann. Rev. Ent., 35 , 421-446.

VIA, s. 1991a. The genetic structure of host plant adaptation in a spatial patchwork: demographic variability among reciprocally transplanted pea aphid clones. Evolution, 45, 827-852.

VIA, s. 1991 b. Variation between strains of the flour beetle Tribolium castaneum in relative performance on five flours. Entomologia exp. appl., 60, 173-182.

VIA, S. AND LANDE, R. 1985. Genotype-environment interaction and the evolution of phenotypic plasticity. Evolution, 39, 505-522.

VIA, S. AND LANDE, R. 1987. Evolution of genetic variability in a spatially variable environment: effects of genotypeenvironment interactions. Genet. Res., 49, 147-156.

YAMADA, Y. 1962. Genotype $\times$ environment interaction and genetic correlation of the same trait under different environments. Jap. J. Genet., 37, 498-509. 